\title{
ESTIMATION DE LA TENEUR EN ACÉTATE DE TRIBUTYLE- ÉTAIN DANS L'EAU DE MER, PAR UNE MÉTHODE BIOLOGIQUE
}

\author{
E. HIS, D. MAURER ET R. ROBERT \\ Institut Scientifique et Technique des Pêches Maritimes \\ 63 Bd Deganne, 33120 Arcachon, France
}

\section{RÉSUMÉ}

L'acétate de tributyle-étain, composé actif de certaines peintures antisalissures, représente un danger certain pour les zones conchylicoles de captage (E. HIS et R. ROBERT, 1980 et R. ROBERT et E. HIS, 1981). Les méthodes chimiques actuelles ne sont pas assez sensibles pour permettre le dosage de ce produit dans l'eau de mer, au seuil de toxicité pour les larves d'huîtres.

Une échelle d'action pour douze concentrations comprises entre $100 \mu \mathrm{g} / \mathrm{l}$. et $0,02 \mu \mathrm{g} / \mathrm{l}$., sur les véligères de Crassostrea gigas est proposée. Elle permet d'évaluer le degrè de contamination de l'eau de mer d'un port de plaisance et son évolution.

\section{ABSTRACT:}

Tributyl tin acetate, an active compound of certain antifouling paints, represents a real danger for oyster farming areas of catching spat (E. HIS et R. ROBERT, 1980 and R. ROBERT et E. HIS, 1981). Current chemical methods are not sensitive enough to guarantee the proportions of this product at the toxicity limits of oyster larvae in sea water. It is proposed that a table of effects should be constructed consisting of concentrations of between $100 \mu \mathrm{g} / \mathrm{l}$. and $0,02 \mu \mathrm{g} / \mathrm{l}$. on the veligeres of Crassostrea gigas. This table enables the degree of pollution and its evolution to be evaluated in the sea water of a harbour.

\section{1-INTRODUCTION:}

Le bassin d'Arcachon se caractérise depuis quelques années par des anomalies tant au niveau de la calcification des huîtres qu'au niveau de la reproduction, les premières étant particulièrement marquées au voisinage des zones portuaires.

Nous avons suspecté l'action des sels organométalliques de l'étain (acétate de tributyleétain: TBT) qui entrent dans la composition de certaines peintures antisallisures. De même FERAL (1982) constate que les anomalies du tractus génital observées chez certains gastéropodes "peuvent être la conséquence d'une pollution liée à la navigation", et
SMITH (1981) met en cause plus précisément les peintures antisalissures dans l'apparition de ces phénomènes.

En ce qui concerne la reproduction naturelle, nous avons mis en évidence dès 1980, le danger que constitue l'utilisation du TBT pour les oeufs et les larves D de Crassostrea gigas, (HIS et ROBERT, 1980 et ROBERT et HIS, 1981) et posé le problème de son interdiction en zone conchylicole.

Des expériences menées par ALZIEU et Coll. (1980) au cours de l'été 1981, ont permis de constater que le TBT "peut à très faibles doses, être une cause des anomalies de la calcification chez l'huître".

Ces faits conjugués ont conduit les pouvoirs publics à interdire l'utilisation de ces peintures antisalissures.

Nous avons cherché dans le présent travail, a déterminer le seuil de sensibilitć des oeufs et des larves de $C$. gigas au TBT.

Par ailleurs, les effets pour des concentrations connues comprises entre $100 \mu \mathrm{g} / \mathrm{l}$. et $0,02 \mu \mathrm{g} / \mathrm{l}$. sont comparés à ceux qui ont été obtenus en été 1981 et au printemps 1982, avec de l'eau de surface prèlevée dans le port de plaisance d'Arcachon soit avant et après ies mesures d'interdiction des peintures à base d'organostanniques. En effet, la détection de ces composés dans l'eau de mer est, à l'heure actuelle, trop peu sensible, pour permettre les dosages au taux d'action de ces produits sur les organismes vivants.

\section{2 - PROTOCOLE EXPERIMENTAL}

Les techniques utilisées ont été exposées antérieurement (HIS et ROBERT, 1981; ROBERT, HIS : MAURER (1982), sous presse). Nous ne ferons qu'tn rappeler les grandes lignes.

On provoque la maturation des huitres dans des bacs en circuit fermé à température de $20^{\circ} \pm 1^{\circ} \mathrm{C}$. Les émissions sont obtenues par choc thermique (passagn répétés de $28^{\circ} \mathrm{C}$ a $15^{\circ} \mathrm{C}$ ). Les élevages, en doubli exemplaire, sont menés dans des béchers stériles contenant deux litres d'eau de mer fraîchement filtric sur membrane SARTORIUS de porosité 0,2 microns. à la température de $24^{\circ} \mathrm{C}$. L'eau de mer est changie 't heures après les fécondations, qui sont réalisèes tn présence des différentes concentrations de TBT, purs 
tous les deux jours, jursqu'a la fin des observations (12ème jour). Chaque changement d'eau s'accompainc d'un renouvellement du micropolluant.

Les vèligères élevées à raison de 8000 individus/litre sont nourries selon les données de HELM et IIII. I ICAN (1977) à l'aide de cultures d'Isochrysis galbana, Chaetoceros calcitrans et Tetraselmis suecica.

Les observations portent sur les pourcentages de larves $\mathrm{D}$ formées, de mortalités et de larves anormales, selon les critères définis par LE PENNEC et LE kOUX (1979), CALABRESE et Coll. (1977); les observations sont effectuées sur 200 larves par elcrage.

La croissance des véligères est étudiée pendan douze jours, par mensuration tous les 2 jours de la hauteur de 50 véligères par élevage, à 1,5 microns près, sur clichés photographiques; les moyennes sont cilculées avec un intervallę de confiance au seuil de securité de $95 \%$.

\section{3 - RESULTATS}

3.1 Rappel des résultats antérieurs.

Ils concernent l'action du TBT sur l'embryogénèse at sur les larves D de Crassostrea gigas à des concentrations comprises entre 100 et $1 \mu \mathrm{g} / \mathrm{l}$, cette dernière valeur se situant déjà en dessous des possibilités de detection des techniques analytiques actuelles.

A un microgramme par litre, toutes les larves D tormées en présence du produit sont anormales; le taux de mortalité est de $100 \%$ en six jours.

Ax-dessus de cette valeur, les larves D ne sont jamais obtenues; les trochophores observées sont loutes aberrantes ( 3 et $5 \mu \mathrm{g} / \mathrm{l}$ ); à $10 \mu \mathrm{g} / \mathrm{l}$., les fecondations se produisent et $80 \%$ des oeufs se segmentent; mäis on n'obtient que de très rares trochophores, toutes monstrueuses. Le nombre d'oeufs segmentés diminue ensuite, de $60 \%(25 \mu \mathrm{g} / \mathrm{l}$.) à $1 \%(50 \mu \mathrm{g} / \mathrm{l}$.). A $100 \mu \mathrm{g} / \mathrm{l}$. les fecondations sont inhibées.

Précisons enfin que l'immersion d'ovules pendant une demi-heure seulement dans une solution de $50 \mu \mathrm{g} / \mathrm{l}$. de TBT, inhibe l'embryogénèse.

3.2. Recherche de la limite de tolérance des larves: nouvelles observations.

Les concentrations suivantes ont été testées: $0 \mu \mathrm{g} / \mathrm{l}$. (témoins), $1 \mu \mathrm{g} / \mathrm{l}$., $0,5 \mu \mathrm{g} / \mathrm{l}$., $0,2 \mu \mathrm{g} / \mathrm{l} ., 0,1 \mu \mathrm{g} /$., $0,05 \mu \mathrm{g} / \mathrm{l} ., 0,02 \mu \mathrm{g} / \mathrm{l}$. Dans tous les cas, les larves D ont été obtenues en 24 heures. Excepté pour la valeur la plus basse, elles présentent des anomalies de la masse viscérale (excroissances débordant des valves fermées) identiques à celles que décrivent CALABRESE et Coll. (1977) qui affectent surtout les élevages à 1 et $0,5 \mu \mathrm{g} / \mathrm{l}$. et disparaissent le second jour. $\mathrm{Ne}$ persistent que les anomalies au niveau de lavéliconche ou du vélum (tableau 1).

De même le régime trophique des véligères est perturbé: le tractus digestif est peu ou pas coloré, dès le second jour à $1 \mu \mathrm{g} / \mathrm{l}$., puis plus tardivement aux autres concentrations (4ème jour à 0,5 et $0,2 \mu \mathrm{g} / 1$.; sixième jour à 0,1 et $0,05 \mu \mathrm{g} / \mathrm{l}$.) certaines larves échappant au phénomène. Seuls les élevages à $0,02 \mu \mathrm{g} / \mathrm{l}$, ne sont que très faiblement affectés le sixième jour.

Les mortalités se stabilisent au-dessous de $10 \%$ dans les élevages témoins et à la plus faible concentration (tableau 2). Dans tous les autres cas, elles sont totales ou subtotales du 6ème au 8ème jour ( 1 et $0,5 \mu \mathrm{g} / \mathrm{l}$.) et au 12 ème.

Tableau 1 - Pourcentages de larves anormales. Les anomalies (m.v.) observées le premier jour concernent la marse viscérale, (présence d'excroissances quand les valves sont fermées). Ce type d'anomalie disparait le second jour. Les valeurs mentionnées ultérieurement concernent soit la véliconche, soit le velum; elles sont restées au mínıe niveau dans tous les cas à partir du deuxième jour.

-+++ perturbation marquée du régime trophique des véligères.

+ faible perturbation du régime trophique des véligères.

Age des larves

exprimé en

jours

1

2

4

6

8
Concentrations exprimées en $\mu g / 1$.

$\begin{array}{ccccc}0,5 & 0,2 & 0,1 & 0,05 & 0,02 \\ \begin{array}{c}95 \\ \text { (m.v.) }\end{array} & \begin{array}{c}15 \\ \text { (m.v.) }\end{array} & \begin{array}{c}11 \\ \text { (m.v.) }\end{array} & \begin{array}{c}14 \\ (\mathrm{~m} . \mathrm{v} .)\end{array} & 4 \\ 18 & 10 & 11 & 5 & 4 \\ 18 & 10 & 11 & 5 & 4 \\ 18 & 10 & 11 & 5 & 4 \\ +++ & +++ & +++ & + & + \\ - & +++ & +++ & +++ & 4 \\ & & & +\end{array}$




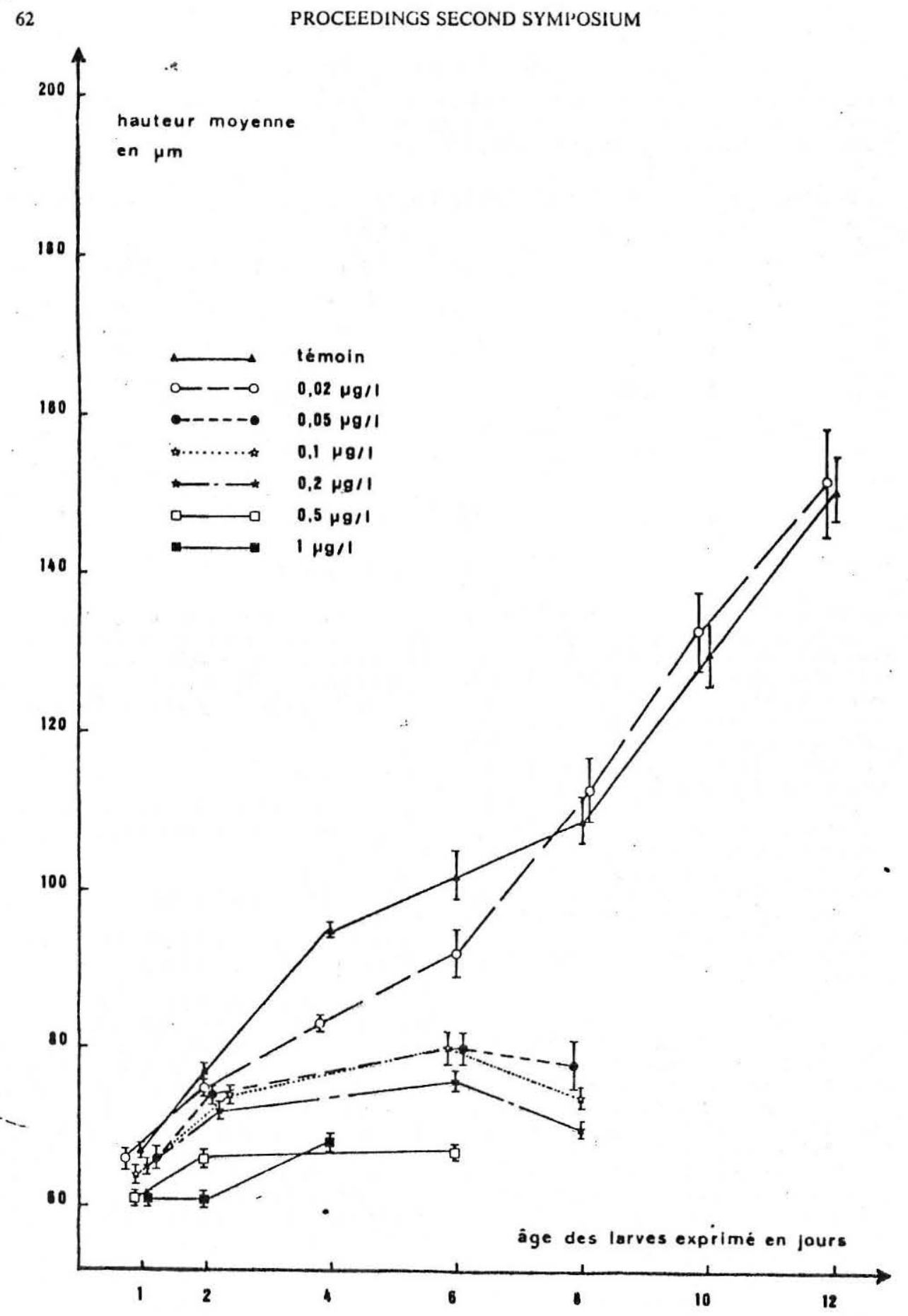

Fig. I - Action de l'acétate de tributyle-étain sur la croissance des larves de Crassosirea gigas. 
Tableau 2 - Pourceńtages de mortalités des véligères de Crassostrea gigas, elevées en eau de l'Ockan, à Jifférentes concentrations d'acétate de tributyle-étain, et en eau du bassin d'Arcachon, prélevée en juillet 1981, dans le chenal du Teychan et dans le port de Plaisance d'Arcachon (Port Arc.).

\begin{tabular}{lcrrrrrrrrr}
$\begin{array}{l}\text { Age des larves } \\
\text { exprimé en } \\
\text { jours }\end{array}$ & \multicolumn{1}{c}{ CONCENTRATIONS EXPRIMEES EN $\mu \mathrm{g} / \mathrm{l}}$. & \multicolumn{3}{c}{$\begin{array}{c}\text { Eau du Bassin } \\
(1981)\end{array}$} \\
1 & 0 & 1 & 0,5 & 0,2 & 0,1 & 0,05 & 0,02 & Teychan Port Arc. \\
2 & 0 & 0 & 0 & 0 & 0 & 0 & 0 & 0 & 0 \\
4 & 1 & 2 & 2 & 2 & 1 & 7 & 1 & 0 & 1 \\
6 & 3 & 25 & 3 & 3 & 1 & 8 & 4 & 4 & 11 \\
8 & 4 & 98 & 14 & 7 & 7 & 8 & 6 & 7 & 11,5 \\
10 & 8 & - & 90 & 15 & 13 & 14 & 6 & 7 & 12 \\
12 & 8 & - & - & 42 & 40 & 60 & 8 & 7 & 14
\end{tabular}

La croissance à $0,02 \mu \mathrm{g} / \mathrm{l}$. est comparable à celle des témoins tableau 3 et figure 1 , malgré un léger ralentissement entre les quatrième et sixième jours qui correspond vraisemblablement à la perturbation du régime trophique.

Dans tous les autres cas, nous observons un talentissement de la croissance qui est fonction de la concentration en TBT, même avant que les mortalités n'interviennent ;il se manifeste très fortement à 1 et $0,5 \mu \mathrm{g} / \mathrm{l}$.

La faible pousse notée entre 0,2 et $0,05 \mu \mathrm{g} / \mathrm{I}$. s'explique par l'existence de quelques véligères dont le regime trophique ne semble pas perturbé, et qui se développent avant d'être décimées par les mortalités: leur nombre augmente dans les élevages quand la concent' .tion en TBT diminue.

3.3 Les élevages en eau du port de plaisance d'Arcachon.

\subsection{1. - Ete 1981.}

De l'eau du port de plaisance a été prélevée le 15 juillet 1981 en surface (ligne de flottaison des cmbarcations), par trois heures de descendant, ainsi yue dans le chenal du Teychan (chenal principal du llassin). Nous comparerons les résultats obtenus dans Ier 2 cas sur les élevages larvaires de $C$.gigas:

- des anomalies de la masse viscérale se thanifestent le second jour $(40 \%)$ en eau du port;

-en 12 jours, la mortalité n'atteint que $7 \%$ en eau

Ju bassin (tableau 2); elle est plus importante en eau

ú port où elle atteint $11 \%$ le 4 ème jour et culmine à

$\$ 10 \%$ en fin d'expériences;

-malgré une hauteur moyenne légèrement supérieure au départ tableau 3 et figure 2 . la -imisance des larves en eau du port est ralentie; en I! Ict la plapart des larves se développent mais on note A persistance de larves $D$ dont la hauteur est pratique" ent stationnaire; il s'en suit une hétérogénéité de la isulation quant à la distribution des tailles, caractéristique d'un milieu perturbé: c'est une des premières manifestations de la présence d'un micropolluant (HIS et ROBERT 1982).

La reprise de croissance, observée du 10ème au 12 ème jour, s'explique par la mortalité de ces véligères de petite taille.

\subsubsection{Printemps 1982 .}

L'eau du port a été prélevée comme précédemment, le 15 avril 1982, après la remise en eau printanière des embarcations:

- à la pleine mer

- par trois heures de descendant

- à la basse mer.

Parallèlement, de l'eau de mer prélevée dans le chenal du Teychan par trois heures de descendant, a été utilisée.

Les pourcentages de mortalités et de larves anormales sont restés faibles dans les quatre cas (inférieurs ou égaux à 20\%), malgré la présence, passagèrement, de quelques véligères présentant des protubérances de la masse viscérale le second jour, en eau du port.

L'examen des courbes de croissance des larves (figure 3 et tableau 3) montre que le développemen des véligères est très semblable dans tous les élevages.

En aucun cas nous n'avons noté des perturbations chez véligères, ou des déséquilibres de populations quant à la distribution des tailles.

\section{4-DISCUSSION}

Sur le plan expérimental, l'action de l'acétate de tributyle-étain, élément èminemment toxique, se manifeste de façon particulièrement marquée aux différentes concentrations testées. Son action progressive a permis d'établir une échelle qui résume 


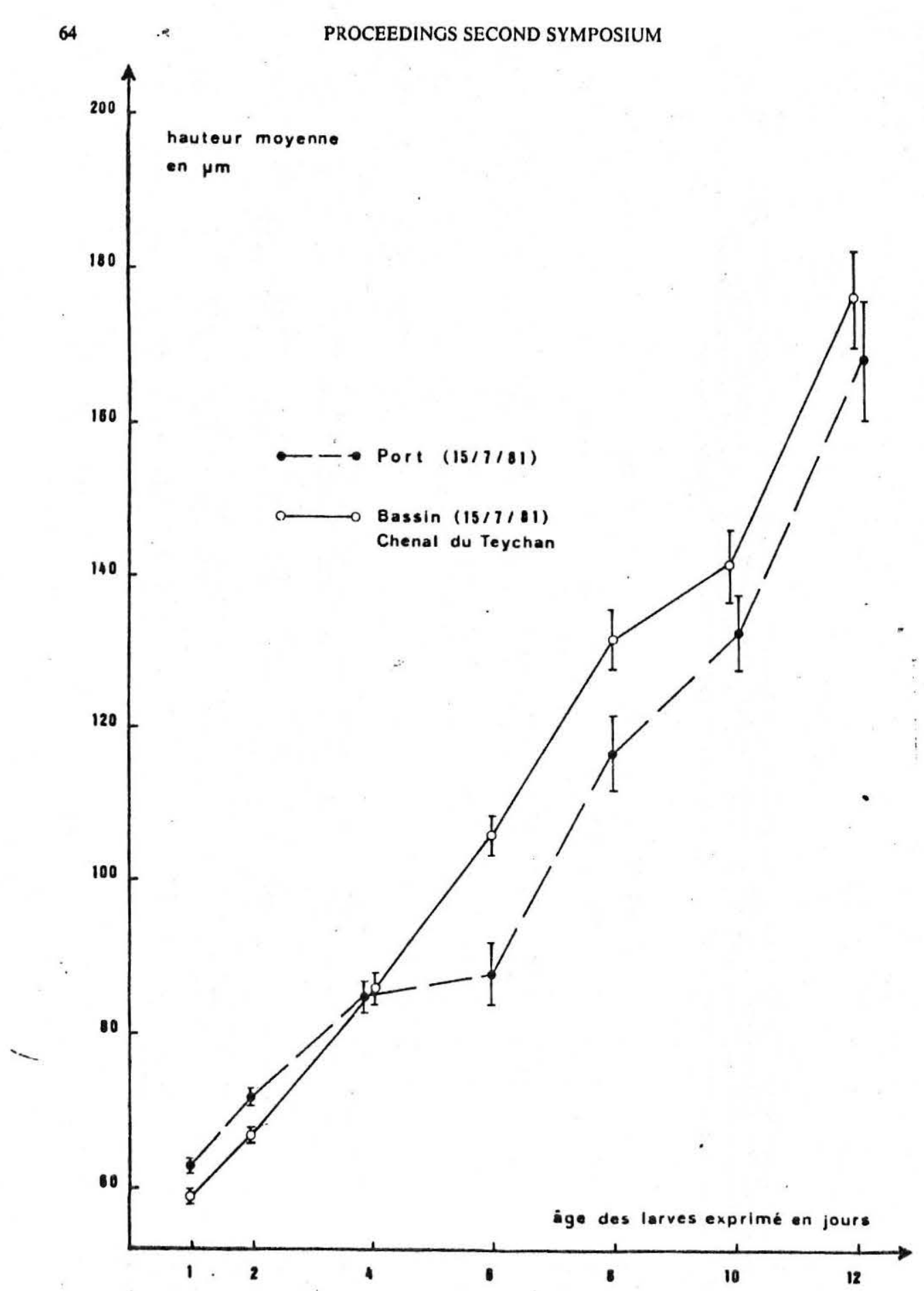

Fig. 2 - Croissance des larves de $C$. gigas èlevees en eau du port de plaisance et en eau du bassin d'Arcachon (Chenal du Teychan), prélevées le 15 juillet 1981. 
Tableau 3 - Hauteurs moyennes exprimées en $\mu \mathrm{m}$ avec intervalle de confiance au seuil de sécurité de $95 \%$, des larves de Crassostrea gigas élevées en eau de l'Océan à différentes concentrations en acétate de tributyle-étain et en eau du bassin d'Arcachon (1981) A: eau prélevée le 15/07/81 dans le chenal du Teychan; B: eau de surface du port de Plaisance à la même date. - 1982: Eaux prélevées au mois d'avril 1982 - C: dans le chenal du Teychan à marée basse. - D :E et F: dans le port de plaisance d'Arcachon respectivement à la pleine mer, par trois heures de descendant et à basse mer.

\section{CONCENTRATIONS en $\mu \mathrm{g} / \mathrm{l}$.}

Age des larves

exprimé en jours

1

2

4

6

8

10

12

0

66,90
$\pm 0,73$

77,07
$\pm 0,71$

$94,77 \quad 68,43$

$\pm 1,18 \quad \pm 0,7$

102,37
$\pm 2,70$

- 67,0

109

109
$\pm 2,7$

$-\quad \pm 0 \overline{85}$

130,14

$\pm 4,00$

150,95
$+4,75$

$0,5 \quad 0,2$

64,04

$+1,46 \quad+2,82$ $\pm 0,87$

0,1

0,05

0,02

60,85

$\pm 0,66$

64,28

65,62

65,62
$\pm 0,76$

66,12
$+0,63$

$\pm 0,63$

74,92

$\pm 0,82$

$\pm 0,65$

$\pm 0,75$

$\pm 0,83$

- 83,14

$\begin{array}{r}83,29 \\ \hline\end{array}$

$75,79 \quad 79,79$

$\pm 1,48$

79,79
$\pm 1,93$

78,82

78,82
$\pm 2,04$

91,92

$\pm 2,84$

$70,49 \quad 73,62$

$77,98 \quad 113,42$

7,31

113,42
$\pm 3,6$

133,31
$\pm 4,49$

151,73
$\pm 7,14$
1981

A B

\section{8,70}

$\pm 0,88$

67,39

$\pm 1,15$

86,45

$\pm 1,91$

105,79
$\pm 2,49$

132,16

132,16
$\pm 5,16$

142,18
$\pm 4,14$

$\pm 6,21$
6265

$\pm 0,69$

71,88

$\pm 0,80$

85,21

$88,31105,31$

$\pm 3,74 \pm 2,51$

$116,71 \quad 134,20$

$\pm 3,25 \quad \pm 3,31$

132,58
$\pm 4,70$

151,45
$\pm 4,25$

169,13
$\pm 2,57$

167,98

$\pm 5,48$
1982

D E

$\begin{array}{lll}67,48 & 65,89 & 67,54\end{array}$ $\pm 0,59 \quad \pm 0,62 \quad \pm 0,62$

$\begin{array}{lll}78,00 & 77,07 & 77,76\end{array}$

$\pm 0,82 \quad \pm 0,79 \quad \pm 0,94$

$88,33 \quad 88,70 \quad 85,99$

$\pm 1,15 \quad \pm 1,04 \quad \pm 1,00$

$108,38 \quad 103,84 \quad 100,03$

$\pm 2,84 \quad \pm 2,38 \quad \pm 2,53$

$\begin{array}{lll}133,49 & 126,05 & 120,39 \text { : }\end{array}$

$\pm 4,13$

$\pm 4,32$

$154,54 \quad 145,31 \quad 144,07$

$\pm 4,19 \quad \pm 4,67 \quad \pm 4,45$

$170,90 \quad 176,14 \quad 161,79$

$\pm 4,35 \quad \pm 5,23 \quad \pm 5,31$ 

l'isentiel de nos observations, depuis le blocage des ficiendations, jusqu'à l'absence d'effet sur la reproduction de Crassostrea gigas (tableau 4).

La comparaison de cés résultats, avec ceux obtenus cll utilisant l'eau de surface du port de plaisance d'Arcachon, permet de situer le degré de contamination de ce dernier:

- au cours du mois de juillet 1981 , l'obtention de larves $\mathrm{D}$ normales, qui présentent pendant la durée des observations une croissance subnormale, permet de situer le seuil au-dessous de la valeur de $0,05 \mu \mathrm{g} / \mathrm{l}$.; les mortalités qui apparaissent les derniers jours, et

Tableau 4 - Echelle d'action de l'acétate de tributyleetain sur la reproduction de Crassostrea gigas.

Concentrations Action sur la reproduction de

$$
(\mu \mathrm{g} / \mathrm{l} \text {.) Crassostrea gigas }
$$

100 Inhibition de la fécondation

50 Inhibition de la segmentation

25 Inhibition partielle de la segmentation $(40 \%)$

10 Absence de formation des trochophores

3 et 5 Pas de véligères - Trochophores monstrueuses

1 Véligères anormales - Mortalité totale en 6 jours

$0,5 \quad$ Nombreuses larves anormales Mortalité totale en 8 jours. Perturbation du régime trophique, s'accentuant du 4ème au 8ème jour. Croissance très réduite.

0,2 Pourcentage de larves $D$ anormales moins élevé. Perturbation du régime trophique dès le 4 ème jour. Mortalités progressives; totales le 12 ème jour. Croissance faible.

0,1 Larves $D$ en majorité normales. Perturbation marquée du régime trophique dès le 6ème jour. Croissance faible jusqu'au 6ème jour. Mortalité subtotale le 12ème jour.

0,05 Larves D normales. Perturbation du régime trophique marquée le 8 ème jour. Mortalités importantes à partir du 10éme jour. Croissance réduite.

0,02 Larves $D$ normales. Mortalités réduites. Bonne croissance. Pas d'action du produit.
I'hétérogénéité des populations larvaires, quant à la distribution des tailles, permettent d'affirmer que l'on se situe au-dessuse de $0,02 \mu \mathrm{g} / \mathrm{l}$.

- au cours du mois d'avril 1982, au contraire, les faibles taux de mortalité et la croissance observée, même à basse mer, permettent d'affirmer que l'eau du port de plaisance contenait dans la fraction dissoute, aux maximum $0,02 \mu \mathrm{g} / \mathrm{l}$. de T.B.T.

\section{CONCLUSIONS}

En l'absence d'une méthode chimique suffisamment fine, les tests de toxicité sur les larves de Crassostrea gigas permettent d'évaluer le degré de contamination d'une eau de mer, en sels organostanniques.

Le temps nécessaire pour obtenir une réponse par cette méthode biologique est de:

-24 heures, pour les concentrations supérieures ou égales à $1 \mu \mathrm{g} / \mathrm{l}$.

-24 heures, entre 1 et $0,5 \mu \mathrm{g} / \mathrm{l}$., avẹc confirmation en six jours.

-8 jours pour les valeurs comprises entre 0,5 et $0,05 \mu \mathrm{g} / \mathrm{l}$.

-10 à 12 jours, pour les valeurs inférieures.

En ce qui concerne le port d'Arcachon, nous avons constaté une diminution de la teneur en T.B.T: de son eau de surface, de l'été 1981 au printemps 1982, pouvant résulter des mesures restrictives prises en début d'année, vis à vis des peintures antisalissures à base de T.B.T.

En ce qui concerne le bassin d'Arcachon, les données obtenues en 1981, avec différentes eaux, sur les larves D nées en écloserie ou prélevées in situ et mises en èlevage (HIS et ROBERT 1981), permettent d'affirmer qu'on se trouvait à un taux égal ou inférieur a $0,02 \mu \mathrm{g} / \mathrm{l}$.

Ceci permet d'exclure la présence de substances susceptibles d'agir sur les larves par toxicité aïgue dans les eaux du bassin d'Arcachon.

\section{BIBLIOGRAPHIE}

ALZIEU, C., THIBAUD, Y., HERAL, M. et BOUTIER, B. 1980. Evaluation des risques dos à l'emploi des peintures anti-salissures dans les zones conchylicoles. Rev. Trav. Inst. Pleches marit. 44 (4), 305-348.

CALABRESES, A. McINNES, J. R., NELSON, D. A. et MILLER, J. E. 1977. Survival and growth of bivalve larvae under heavy metals stress. - Bull. Environn. Contam. Toxicol. 11 (1), 92-97.

FERAL, C. 1982. Etude experimentale des mécanismes assurant l'apparition, le maintien et le cycle d'un tractus génital mâle externe chez les femelles de Nucella lapillus (L). Nassarius reticulatus (L) Ocenebra erinapea (L) (L), Nassarius rencularus (L), Ocenebra erinacea (L) Moil Nat., Caen, $183 \mathrm{p}$.
Sci. Nonochoriques. These de

HELIM, M. M. et MILLICAN, P. F. 1977. Experiments in the hatchery rearing of Pacific oyster larvae (Crassostrea gigas Thunberg). Aquaculture, 11, 1-12.

HIS, E. et ROBERT R. 1980. Action d'un sel organo-mettallique, l'acétate de tributyl-étain sur les oeufs et les larves D de Crassostrea gigas (Thunberg). Cons. inter. Explor. Mer, Comité de la Mariculture, C. M. 1980/F, 27, 10p.

HIS, E. et ROBERT, R. 1981. Les causes de mortalités larvaires de Crassostrea gigas dans le bassin d'Arcachon. Rapport I.S.T.P.M., 7 Septembre 1981, 31p. + annexes. 


\section{PROCEEDINGS SECOND SYMPOSIUM}

HIS, E. et ROBERT, R. 1982. - Le danger de traitement par le sulfate de cuivre en zone conchylicole: toxicitè vis-à-vis des oeufs et des jeunes larves de Crassostrea gigas, Rev. Trav. Inst. Pêches marit., $\mathbf{4 5}(2), 117-125$.

LUCAS, A., LE PENNEC, M., PRIEUR, D. el LEROUX, S 1976. Elevages expérimentaux de larves de mollusques marins. Laboratoire de Zoologie, Aquaculture e Pollutions Marines, Brest., 12 p.

LE PENNEC, M. et LE ROUX, S. 1979. Effets d'u pétrole brut sur la formation de la coquille de Mytilus edulis (L.) (Mytilidae, Bivalvia). Rev. Inst. Océanogr. Med., 55, 49-55.

ROBERT, R. et HIS, E. 1981. Action de l'acétate de tributyle étain sur les oeufs et les larves D de deux molusque d'interêt commercial: Crassostrea gigas (Thunberg) Mytilus galloprovincialis (Lmk) Cons. intern. Explor mer. Comite de la Mariculture, C. M. Molif: 42, is p. ROBERT, R., HIS, E. et MAURER, D 1982. L'unité d'éco physiologie et de Molysmologie larvaire des bivalve d'intérêt commercial du laboratoire d'Arcachon., Science et Pêche, sous presse.

MITH, B. S. 1981. Tributyltin compounds induce male char acteristics on female mud snails Nassarius obsoletus: Ilyanassa obsoleta. - J. Appl. Toxicol. 1 (3), 141-144 